\title{
Crystal electric field excitations in the quantum spin liquid candidate $\mathrm{NaErS}_{2}$
}

\author{
Shang Gao, ${ }^{1, *}$ Fan Xiao, ${ }^{2,3, \dagger}$ Kazuya Kamazawa, ${ }^{4}$ Kazuhiko Ikeuchi, ${ }^{4}$ Daniel Biner, ${ }^{3}$ \\ Karl W. Krämer ${ }^{3}{ }^{3}$ Christian Rüegg, ${ }^{5,6,7,8}$ and Taka-hisa Arima ${ }^{1,9}$ \\ ${ }^{1}$ RIKEN Center for Emergent Matter Science, Wako 351-0198, Japan \\ ${ }^{2}$ Laboratory for Neutron Scattering and Imaging, Paul Scherrer Institut, CH-5232 Villigen PSI, Switzerland \\ ${ }^{3}$ Department of Chemistry and Biochemistry, University of Bern, Freiestrasse 3, Bern, Switzerland \\ ${ }^{4}$ Neutron Science and Technology Center, Comprehensive Research Organization for Science and Society, Tokai, Ibaraki, 319-1106, Japan \\ ${ }^{5}$ Neutrons and Muons Research Division, Paul Scherrer Institut, CH-1211 Villigen PSI, Switzerland \\ ${ }^{6}$ Department of Quantum Matter Physics, University of Geneva, CH-1211 Geneva, Switzerland \\ ${ }^{7}$ Institute for Quantum Electronics, ETH Zürich, CH-8093 Zürich, Switzerland \\ ${ }^{8}$ Institute of Physics, École Polytechnique Fédérale de Lausanne, CH-1015 Lausanne, Switzerland \\ ${ }^{9}$ Department of Advanced Materials Science, University of Tokyo, Kashiwa 277-8561, Japan
}

(Received 24 November 2019; revised 30 May 2020; accepted 30 June 2020; published 16 July 2020)

\begin{abstract}
The delafossite family of compounds with a triangular lattice of rare earth ions has been recently proposed as a candidate host for quantum spin liquid (QSL) states. To realize QSLs, the crystal electric field (CEF) ground state of the rare earth ions should be composed of a doublet that allows sizable quantum tunneling, but until now the knowledge on CEF states in the delafossite compounds is still limited. Here we employ inelastic neutron scattering (INS) to study the CEF transitions in a powder sample of the delafossite $\mathrm{NaErS}_{2}$, where the large total angular momentum $J=15 / 2$ of the $\mathrm{Er}^{3+}$ ions and the resulting plethora of CEF transitions enable an accurate fit of the CEF parameters. Our study reveals nearly isotropic spins with large $J_{z}= \pm 1 / 2$ components for the $\mathrm{Er}^{3+} \mathrm{CEF}$ ground states, which might facilitate the development of a QSL state. The scaling of the obtained CEF Hamiltonian to different rare earth ions suggests that sizable $J_{z}= \pm 1 / 2$ components are generally present in the CEF ground states, supporting the ternary sulfide delafossites as potential QSL hosts.
\end{abstract}

DOI: 10.1103/PhysRevB.102.024424

\section{INTRODUCTION}

The QSL state, where the conventional magnetic longrange order (LRO) is completely removed by quantum fluctuations, has been fascinating physicists since it was proposed in the 1970s [1]. Similar to the well-known cases of onedimensional spin chains [2], the fundamental excitations in QSLs are fractional spin-1/2 excitations called spinons, which can be either gapped or gapless depending on the specific system [3-6]. Theoretical investigations have revealed the spinons in some QSLs to be highly entangled with each other, leading to fractional statistics and exotic braiding properties that might be utilized for topological quantum computing $[7,8]$.

The initial quest for QSLs was focused on intrinsic spin-1/2 systems such as the $\mathrm{Cu}^{2+}$-based compounds [6,9]. One prominent example is the herbertsmithite $\mathrm{ZnCu}_{3}(\mathrm{OH})_{6} \mathrm{Cl}_{2}$ [10-12]. In this compound, the $\mathrm{Cu}^{2+}$ ions form a two-dimensional (2D) kagomé lattice with geometric frustration. Using INS, an almost featureless excitation continuum was revealed [11], which is consistent with the spinon excitations and, more importantly, demonstrates that QSL can exist in real materials.

* Present address: Materials Science \& Technology Division and Neutron Science Division, Oak Ridge National Laboratory, Oak Ridge, Tennessee 37831, USA; shang.gao@ riken.jp

${ }^{\dagger}$ fan.xiao@psi.ch
Recently, the search for QSL candidates has been extended to the rare earth systems. This is surprising at first glance, because the rare earth ions usually have a relatively large angular momentum $J$, which disfavors quantum fluctuations. However, with an appropriate CEF, the ground state doublet of the rare earth ions might have considerable components of $\left|J, J_{z}\right\rangle$ with a relatively small $\left|J_{z}\right|$ that allows quantum tunneling $[13,14]$. If this ground state is well separated from the excited states, the spin degree-of-freedom of the rareearth ions will effectively behave as spin- $1 / 2$. One of the best-known examples is the quantum spin ice state realized in the rare-earth pyrochlores $[15,16]$. For the Dy and Ho-based pyrochlore systems with only relatively large $\left|J_{z}\right|$ components in the CEF ground state, a classical spin ice state is realized, where each tetrahedron has a two-in-two-out spin configuration $[17,18]$. While for the $\mathrm{Tb}, \mathrm{Yb}$, and Pr-based pyrochlores [19-21], a relatively high magnitude of quantum spin tunnellings is observed, which drives the classical spin ice state into a QSL state with emergent U(1) quantum electrodynamics [15,22].

Given the success of the effective spin-1/2 picture in the rare-earth pyrochlores, it is natural and tempting to advance this concept to other frustrated lattices, especially the 2D triangular lattice where the idea of QSL was originally conceived [1]. According to theoretical calculations [23], the effective spin-1/2 Hamiltonian for rare earth spins on a triangular lattice might contain transverse coupling terms that can induce competing ground states in the classical 
(a)

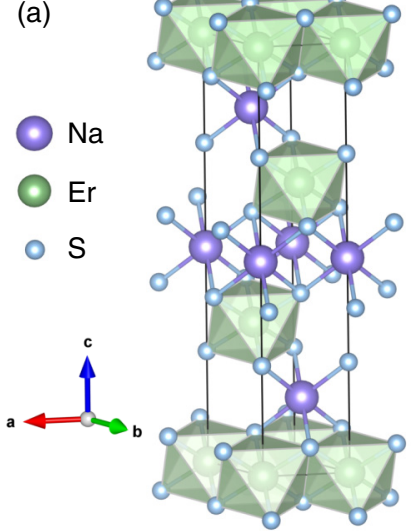

(b)

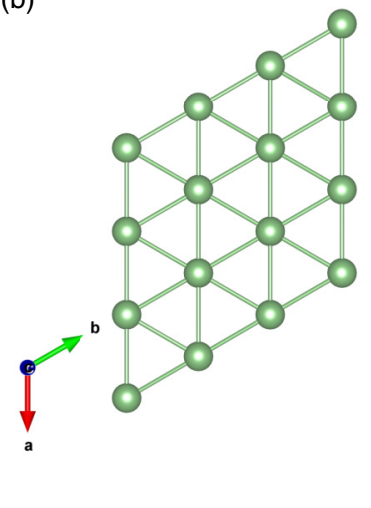

FIG. 1. (a) Crystal structure of $\mathrm{NaErS}_{2}$ [30]. The $\mathrm{Na}^{+}$and $\mathrm{Er}^{3+}$ ions occupy the $3 b$ and $3 a$ sites, respectively. The $\operatorname{ErS}_{6}$ octahedra are explicitly shown. (b) The triangular lattice formed by the $\mathrm{Er}^{3+}$ ions viewed along the $c$ axis.

solution, whereupon a QSL state could emerge near the phase boundary once quantum fluctuations are included. Following this argument, the triangular lattice compound $\mathrm{YbMgGaO}_{4}$ has recently been proposed as a candidate host for the QSL state [24-26]. However, due to the $\mathrm{Mg}$-Ga disorder that is intrinsic in this compound, it is unclear whether the broad excitations that have been observed in INS are due to quantum fluctuations or disorder effects [27-29].

The delafossite family of compounds $A \operatorname{Ln} X_{2}$, where $\operatorname{Ln}$ are rare earth ions, $A=\mathrm{Na}, \mathrm{K}, \mathrm{Cu}(\mathrm{I})$, and $X=\mathrm{O}, \mathrm{S}, \mathrm{Se}$, might be the sought-after QSL candidates that are free from any disorder [31-34]. Similar to the parent delafossite mineral $\mathrm{CuFeO}_{2}, A \operatorname{Ln} X_{2}$ crystallizes in the space group $R \overline{3} m$ [30], with both $A$ and $\mathrm{Ln}$ sites forming triangular lattices as shown in Fig. 1. Especially, the $\operatorname{Ln} X_{2}$ layers consist of $\operatorname{Ln}$ ions with $D_{3 d}$ site symmetry located at the center of edge-sharing $X$-octahedra, similar to $\mathrm{YbMgGaO}_{4}$. Detailed experimental studies on the magnetic properties of rare earth delafossites have been reported for the Yb-based compounds, including $\mathrm{NaYbS}_{2}$ [35,36] and $\mathrm{NaYbO}_{2}$ [37-39], which revealed the absence of magnetic LRO in both compounds and suggested possible QSL states.

In order to facilitate the QSL search in the delafossites, it is crucial to have an overview of their CEF environment. As exemplified by the spin ice compounds [40-43], the CEF parameters in systems with similar structures normally obey the scaling rule. Therefore, compared to the $\mathrm{Yb}^{3+}$ ions with $J=7 / 2[35,39]$, rare earth ions with a larger $J$ allow more CEF transitions, which will enable a more accurate fit of the CEF parameters and thus provide a reference in the study of the similar delafossite compounds.

Here we report INS investigations on the CEF transitions in $\mathrm{NaErS}_{2}$, where the $\mathrm{Er}^{3+}$ has a total angular momentum of $J=15 / 2$. Our studies reveal nearly isotropic spins with large $J_{z}= \pm 1 / 2$ components for the $\mathrm{Er}^{3+}$ CEF ground state doublet that allow spin quantum tunneling $[13,14]$. The scaling of the obtained CEF Hamiltonian to different rare earth ions will foster the search for QSL states in the sulfide delafossites.

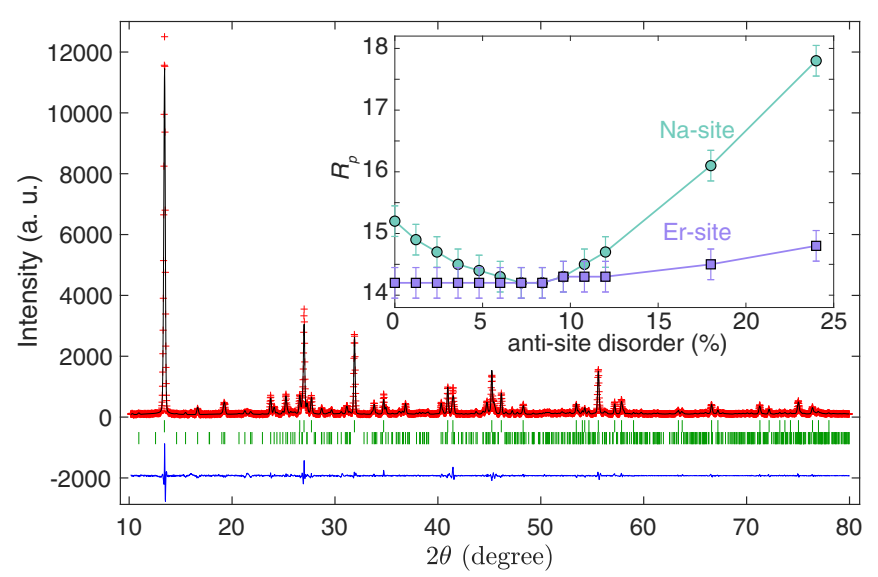

FIG. 2. Refinement results of the x-ray diffraction data measured at room temperature for $\mathrm{NaErS}_{2}$ polycrystalline sample. Data points are shown as red crosses. The calculated pattern is shown as the black solid line. The upper and lower vertical bars show the positions of the Bragg peaks for $\mathrm{NaErS}_{2}$ and $\mathrm{Er}_{2} \mathrm{~S}_{3}$, respectively. The blue line at the bottom shows the difference of measured and calculated intensities. Inset shows the $R_{p}$ factor as a function of the antisite disorder at the $\mathrm{Na}$ and Er sites.

\section{EXPERIMENTAL DETAILS}

Polycrystalline samples of $\mathrm{NaErS}_{2}$ were prepared using the solid state method proposed by Schleid et al. [30], in which $\mathrm{NaCl}$ served as both reagent and flux. Under $\mathrm{N}_{2}$, Er grains, sulfur, and $\mathrm{NaCl}$ in a molar ratio of 2:3:9 were loaded into a $\mathrm{Ta}$ ampoule, which was sealed by arc welding under He. The Ta ampoule was sealed in a silica ampoule under vacuum, slowly heated up to $850^{\circ} \mathrm{C}$ with $20^{\circ} \mathrm{C} / \mathrm{h}$ and kept for seven days before cooling down to room temperature. The final product was rinsed with $\mathrm{H}_{2} \mathrm{O}$ and acetone several times to remove water-soluble $\mathrm{Na}_{3} \mathrm{ErCl}_{6}$.

Powder X-ray diffraction (XRD) patterns were measured on a STOE STADIP diffractometer in reflection (BraggBrentano) geometry in air at room temperature. Diffraction patterns with $\mathrm{Cu} K_{\alpha 1}$ radiation $(\lambda=1.54059 \AA)$ from a focusing $\alpha-\mathrm{SiO}_{2}$ (101) monochromator were recorded on a linear position-sensitive detector with $0.01^{\circ}$ resolution in $2 \theta$. Rietveld refinement was performed in the $R \overline{3} \mathrm{~m}$ space group using the FULLPROF program [44].

INS experiments were performed on the 4SEASONS timeof-flight (TOF) spectrometer at the Materials and Life Science Experimental Facility MLF of J-PARC in Japan [45]. The setup with a radial collimator and a neutron beam size of $20 \times 20 \mathrm{~mm}^{2}$ was employed. A $\mathrm{NaErS}_{2}$ powder sample of $1.8 \mathrm{~g}$ was packed in an envelope of aluminum foil, curled up and installed in an aluminium sample can with outer/inner diameter of $20.5 / 20.0 \mathrm{~mm}$. This configuration reduced the neutron absorption caused by the Er isotopes in the sample $[42,46]$. For our measurements, the chopper frequency was set to $300 \mathrm{~Hz}$, and the repetition rate multiplication method [47] allows the measurement with multiple incident energies of $E_{i}=222,80,41,24.7,16$, and $12 \mathrm{meV}$ to be collected at the same time. A GM refrigerator was mounted to reach temperatures between 5 and $250 \mathrm{~K}$. Besides the $\mathrm{NaErS}_{2}$ sample, measurements were also performed on a 


\section{(a)}
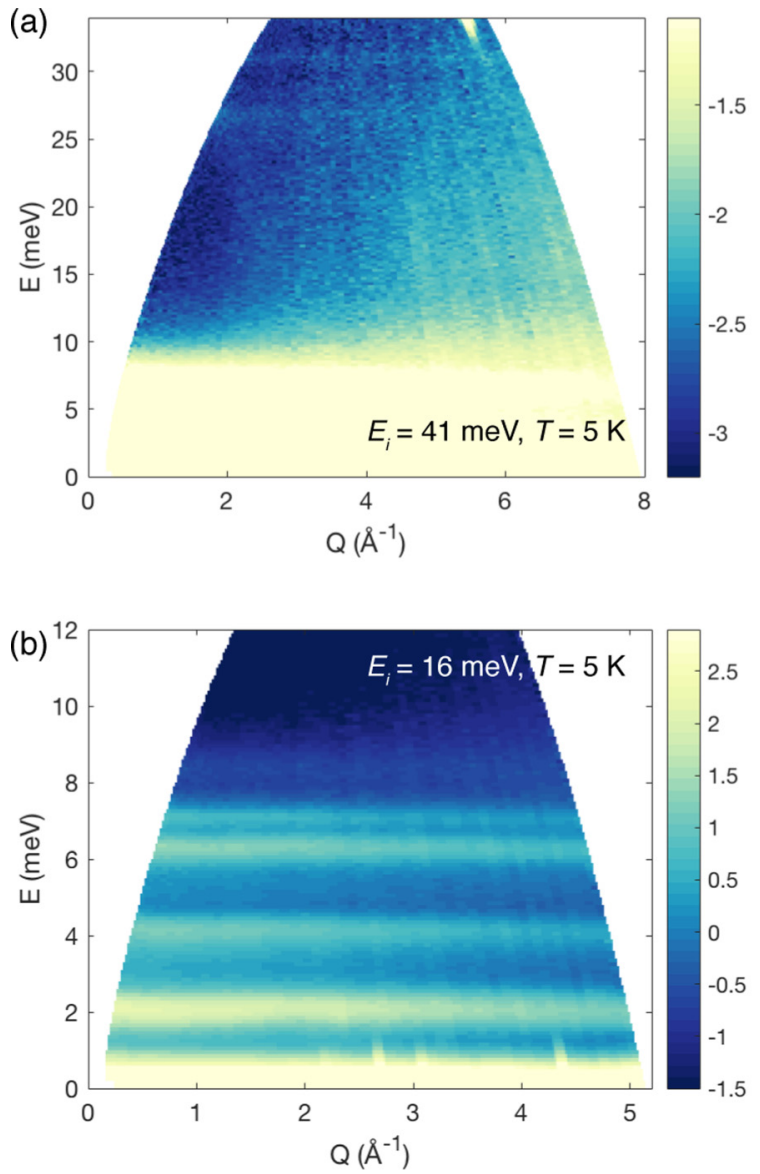
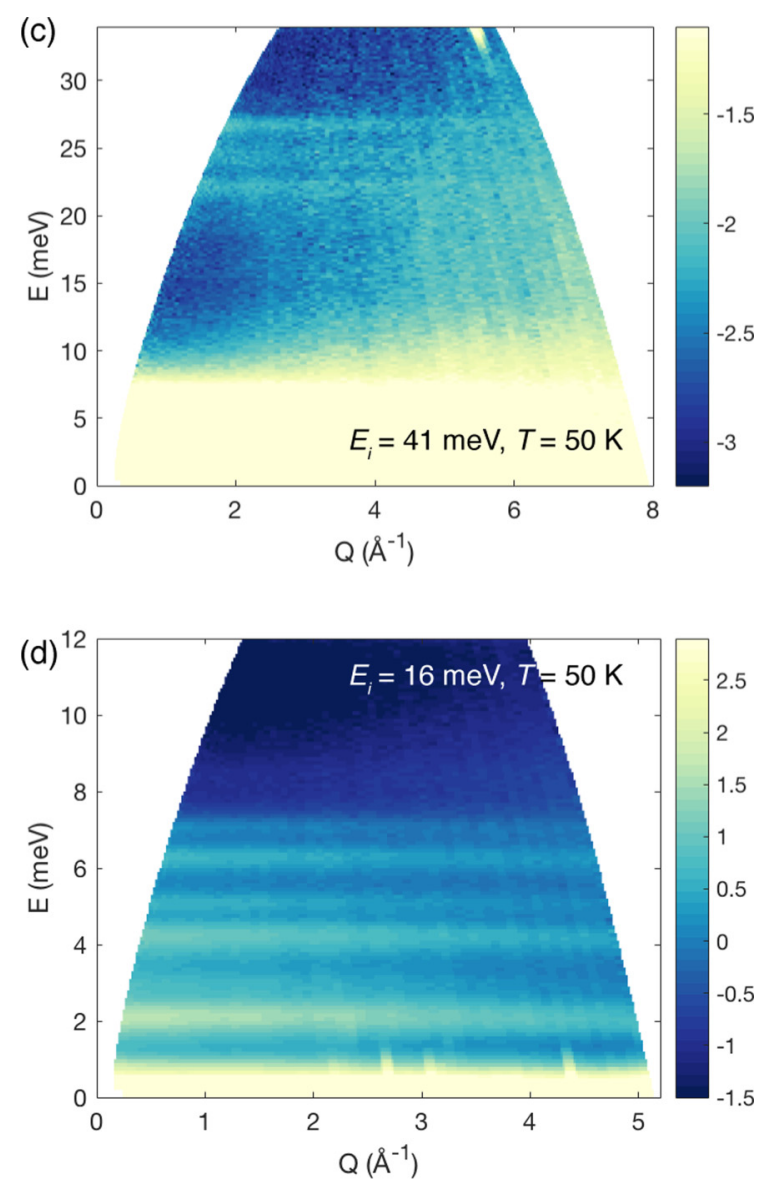

FIG. 3. INS spectra $S(Q, \omega)$ of a $\mathrm{NaErS}_{2}$ powder sample collected on 4 SEASONS at temperatures $T=5 \mathrm{~K}[(\mathrm{a})$ and (b)] and $50 \mathrm{~K}[(\mathrm{c})$ and (d)], with incoming neutron energy $E_{i}=16 \mathrm{meV}[(\mathrm{b})$ and (d)] and $41 \mathrm{meV}$ [(a) and (c)]. At elevated temperatures, additional excitations originating from thermally populated doublets are observed.

vanadium standard to allow a quantitative comparison for data collected at $300 \mathrm{~K}$ with the same instrumental setup. The acquired data were analyzed with the UTSUSEMI software package [48].

\section{RESULTS}

The XRD pattern for our $\mathrm{NaErS}_{2}$ sample is shown in Fig. 2. The refined lattice parameters $a=3.93343(4) \AA$ and $c=19.8378(2) \AA$ are in good agreement with the published crystal structure [30]. $\mathrm{NaErS}_{2}$ crystallizes in space group $R \overline{3} \mathrm{~m}$ with $\mathrm{Na}^{+}$and $\mathrm{Er}^{3+}$ ions on sites $3 b\left(\begin{array}{lll}0 & 0 & 0.5\end{array}\right)$ and $3 a\left(\begin{array}{ll}0 & 0\end{array}\right.$ $0)$, respectively. The $S^{2-}$ ions occupy the site $6 c\left(\begin{array}{lll}0 & 0 z\end{array}\right)$ with $z=0.2461(4)$. A satisfactory fit was obtained by including a preferred orientation along the [001] direction due to the platelike habit of the $\mathrm{NaErS}_{2}$ polycrystals. The $R$ factors are $R_{p}=14.2 \%, R_{w p}=15.8 \%$, and $\chi^{2}=1.9$. The inset of Fig. 2 presents the value of the $R_{p}$ factor as a function of the antisite disorder at the $\mathrm{Na}$ and $\mathrm{Er}$ sites. Although the $\mathrm{Er}$ sites are fully occupied within our experimental resolution, a small fraction of $7 \%$ antisite disorder is discerned at the $\mathrm{Na}$ sites. This disorder on the Na sites might cause the taillike broadening in the CEF excitations as discussed in the following section.
A secondary phase is observed in all the synthesized batches, which can be assigned to the $\mathrm{Er}_{2+x} \mathrm{~S}_{3+y}$ impurities and has been treated with the Le Bail profile fit assuming a $P 2_{1} / m$ space group. Using the strongest reflections for $\mathrm{NaErS}_{2}$ at $2 \theta \sim 13^{\circ}$ and for the secondary phase at $2 \theta \sim$ $25^{\circ}$, the fraction of the secondary phase is estimated to be $\sim 5 \%$.

Figure 3 summarizes the $\mathrm{NaErS}_{2}$ neutron spectra collected at $T=5$ and $50 \mathrm{~K}$ with $E_{i}=16$ and $41 \mathrm{meV}$. The strong intensity spot in the $E_{i}=41 \mathrm{meV}$ spectra at wave-vector transfer $Q=5.5 \AA^{-1}$ and energy transfer $E=34 \mathrm{meV}$ is spurious due to unshielded scattered neutrons from the beam catcher. For TOF neutron spectrometers, the energy resolution scales with the incoming neutron energy and can be estimated by the full width at half maximum (FWHM) of the incoherent scattering in the vanadium standard measurements. In our experiment, the energy resolution was estimated to be 0.50 , 0.80 , and $2.51 \mathrm{meV}$ for $E_{i}=12,16$, and $41 \mathrm{meV}$, respectively. Therefore, a relatively high $E_{i}$ of $41 \mathrm{meV}$ allows access to the high energy excitations, while a relatively low $E_{i}$ of 16 or $12 \mathrm{meV}$ resolves the different excitations at low energies.

At $T=5 \mathrm{~K}$, four dispersionless excitations are observed at around 2.0, 4.0, 6.0, and $6.8 \mathrm{meV}$ in the $E_{i}=16 \mathrm{meV}$ spectra shown in Fig. 3(b), and three relatively weak excitations can be discerned at $26.5,28.3,30.9 \mathrm{meV}$ in the $E_{i}=41 \mathrm{meV}$ 

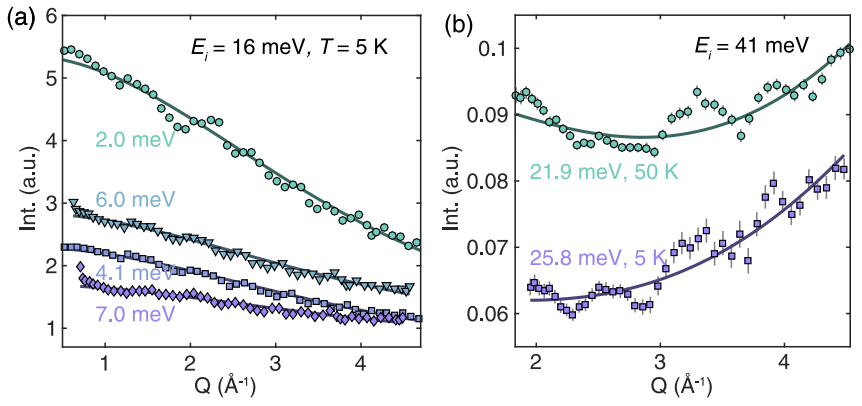

FIG. 4. (a) Momentum transfer dependence of the CEF excitations with $E_{i}=16 \mathrm{meV}$ at $T=5 \mathrm{~K}$. Data points represent intensities integrated within an energy range of $1.2 \mathrm{meV}$ centered around $2.0 \mathrm{meV}$ (green circles), $4.1 \mathrm{meV}$ (purple squares), $6.0 \mathrm{meV}$ (green triangles), and $7.0 \mathrm{meV}$ (purple diamonds). Solid lines are the corresponding fits by the magnetic form factor of the $\mathrm{Er}^{3+}$ ions plus a flat background to confirm the magnetic origin of the excitations. (b) Comparison for the momentum transfer dependence of the CEF excitations with $E_{i}=41 \mathrm{meV}$. Purple squares (green circles) are intensities integrated within an energy range of 2.5 (1.6) meV centered around 25.8 (21.9) meV measured at 5 (50) K. Solid lines are the corresponding fits by the magnetic form factor of the $\mathrm{Er}^{3+}$ ions plus a flat background together with a $Q^{2}$ term.

spectra shown in Fig. 3(a). In $D_{3 d}$ symmetry, the $\mathrm{Er}^{3+}{ }^{4} I_{15 / 2}$ manifold splits into eight Kramers doublets. Therefore, the seven excitations observed in our INS spectra can be ascribed to the Stokes transitions from the CEF ground state doublet to the seven excited doublets. At an elevated temperature of $50 \mathrm{~K}$, the excited doublets are thermally populated, leading to two additional transitions at $\sim 2.9$ and $5.0 \mathrm{meV}$ in Fig. 3(d) and three high-energy transitions at 22.0,24.0, and $26.5 \mathrm{meV}$ in Fig. 3(c). Transitions at $\sim 2.9,22.0,24.0$, and $26.5 \mathrm{meV}$ are due to the excitations from the doublet at $\sim 2.0 \mathrm{meV}$, while the transition at $\sim 5.0 \mathrm{meV}$ is due to the excitations from the doublet at $\sim 4.0 \mathrm{meV}$.

INS probes the CEF transitions though the dipolar interactions between the neutron and electron spins [see Eq. (2)]. Therefore, the neutron scattering length for CEF transitions should be proportional to the magnetic form factor $f(Q)$ of the $\mathrm{Er}^{3+}$ ions that is monotonously decreasing with $Q$. Figure 4(a) plots the $Q$ dependence of the INS intensities integrated at around $E=2.0,4.1,6.0$, and $7.0 \mathrm{meV}$ within an energy width of $1.2 \mathrm{meV}$. The integrated intensities decrease monotonously with $Q$ and can be fitted by the square of the form factor $f^{2}(Q)$ plus a constant background, which confirms the CEF origin of these excitations. In contrast, the high- $E$ modes observed at $T=5 \mathrm{~K}$ shown in Fig. 4(b) exhibit a $Q$-quadratic behavior that is typical for phonon excitations. At $50 \mathrm{~K}$, the CEF contributions to the $4.0 \mathrm{meV} \rightarrow 25.8 \mathrm{meV}$ transition become more obvious, leading to a nonmonotonous $Q$ dependence for the 21.9-meV mode that can be described by the $\mathrm{Er}^{3+}$ form factor plus a $Q^{2}$ term. Therefore, the high- $E$ modes should have contributions from both the CEF and phonon excitations.

The energies of the CEF levels together with their INS intensities can be quantitatively analyzed using the CEF Hamiltonian. As noted by Hutchings in the 1960s [49], different normalization schemes exist for the CEF operators, leading to different conventions in the CEF Hamiltonian definition.
TABLE I. Fitted Wybourne CEF parameters (meV) for $\mathrm{Er}^{3+}$ in $\mathrm{NaErS}_{2}$. Errors are conservative estimates based on repeated Monte Carlo simulations.

\begin{tabular}{lccccc}
\hline \hline$L_{2}^{0}$ & $L_{4}^{0}$ & $L_{4}^{3}$ & $L_{6}^{0}$ & $L_{6}^{3}$ & $L_{6}^{6}$ \\
\hline$-24.7(1)$ & $-76.8(4)$ & $-128.0(7)$ & $29.3(1)$ & $-0.1(1)$ & $24.6(1)$ \\
\hline \hline
\end{tabular}

In the Stevens convention, the Hamiltonian is usually written as $\mathcal{H}=\sum_{l, m} B_{l}^{m} \hat{O}_{l}^{m}$, where the normalization factors for the CEF operators $\hat{O}_{l}^{m}$, or the so-called Stevens factors, are implicitly included in the CEF parameters $B_{l}^{m}$. Here the integer $l$ ranges from 0 to 6 for $f$ electrons, and the integer $m$ ranges from $-l$ to $l$. In the Wybourne convention, the Hamiltonian can be written as $\mathcal{H}=\sum_{l, m} L_{m}^{l} \hat{C}_{m}^{l}$, with the Stevens factors included in the CEF operators $\hat{C}_{m}^{l}$ instead of the CEF parameters $L_{m}^{l}$. Here we follow the Wybourne convention and introduce the CEF operators $\hat{T}_{l}^{m}=\hat{C}_{-m}^{l}+(-1)^{m} \hat{C}_{m}^{l}$ for $m \geqslant$ 0 as implemented in the McPhase program [50]. For rare-earth ions with $D_{3 d}$ symmetry, the CEF Hamiltonian becomes:

$$
\mathcal{H}=L_{2}^{0} \hat{T}_{2}^{0}+L_{4}^{0} \hat{T}_{4}^{0}+L_{4}^{3} \hat{T}_{4}^{3}+L_{6}^{0} \hat{T}_{6}^{0}+L_{6}^{3} \hat{T}_{6}^{3}+L_{6}^{6} \hat{T}_{6}^{6},
$$

where the $z$ direction is along the threefold rotation axis. The CEF parameters thus defined are related to the original Wybourne CEF parameters by a factor of $(-1)^{m}$.

Due to the large separation of $\sim 800 \mathrm{meV}$ between the lowenergy manifold ${ }^{4} I_{15 / 2}$ and the higher-energy manifolds for isolated $\mathrm{Er}^{3+}$ ions, we diagonalize the CEF Hamiltonian in the Hilbert space spanned by the basis vectors $\left|J=15 / 2, J_{z}\right\rangle$ within the ${ }^{4} I_{15 / 2}$ manifold. The INS cross section for the CEF excitations on a powder sample is then expressed as [51,52]

$$
\frac{d^{2} \sigma}{d \Omega d E}=c f^{2}(Q) \frac{k_{f}}{k_{i}} \sum_{\alpha} \sum_{i, f} p_{i}\left|\left\langle f\left|\hat{J}_{\alpha}\right| i\right\rangle\right|^{2} \delta\left(E_{i}-E_{f}+E\right) \text {, }
$$

where $c$ is a constant, and $|i\rangle$ and $|f\rangle$ are the eigenfunctions of the CEF Hamiltonian and represent the initial and final wave functions, respectively. $E_{i}\left(k_{i}\right)$ and $E_{f}\left(k_{f}\right)$ are the energies (wave vectors) of the incoming and scattered neutrons, respectively. The occupation probability $p_{i}$ for the state at $E_{i}$ is described by the Boltzmann distribution $p_{i}=$ $\exp \left(-E_{i} / k T\right) / \sum_{i} \exp \left(-E_{i} / k T\right) . \hat{J}_{\alpha}$ with $\alpha=x, y$, and $z$ are the angular momentum operators. $\delta\left(E_{i}-E_{f}+E\right)$ is the delta function.

By combining the SAFiCF code [53] with the particle swarm optimization algorithm, we can fit the INS spectra by varying the CEF parameters. Calculations were also checked using the McPhase program [50]. Figure 5 plots the energy dependence of the INS intensities integrated within a momentum transfer of $1.2 \sim 2.2(2.2 \sim 3.2) \AA^{-1}$ for the $E_{i}=12$ (41) $\mathrm{meV}$ data measured at $T=5$ and $50 \mathrm{~K}$, respectively. The calculated spectra are convoluted by a Gaussian function to account for the instrument resolution. The best fit is achieved with the set of CEF parameters shown in Table I. The taillike broadening of the CEF excitations on the lower- $E$ side in Fig. 5(a) might be related to the disorder at the $\mathrm{Na}$ sites as observed in our XRD refinement. The slight mismatch for the $E_{i}=41 \mathrm{meV}$ data shown in Figs. 5(b) and 5(d) might be due to the CEF-phonon hybridization as revealed from 

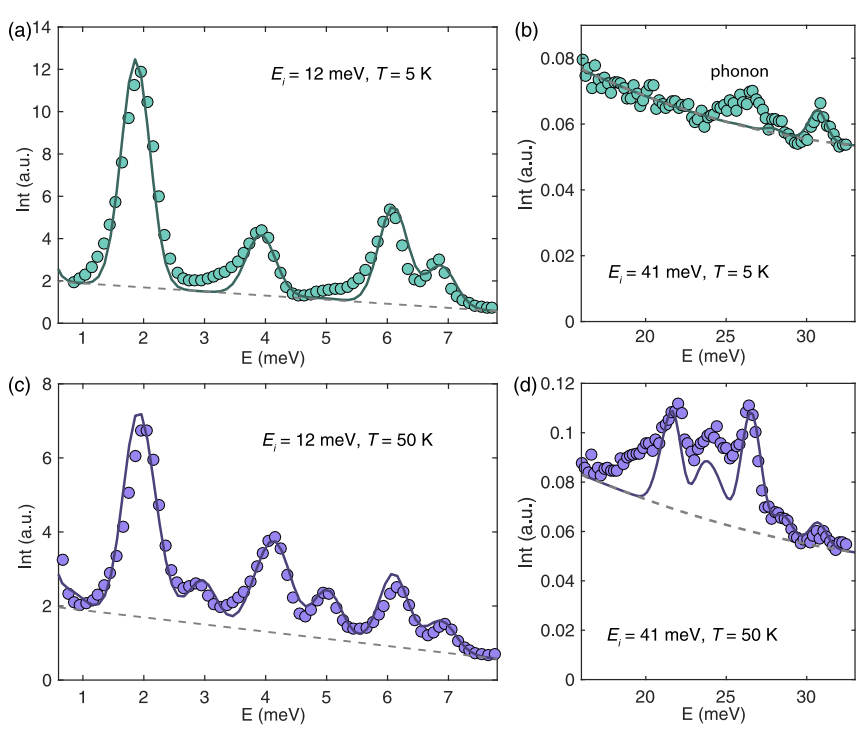

FIG. 5. CEF excitations collected at $T=5 \mathrm{~K}[(\mathrm{a})$ and (b)] and $50 \mathrm{~K}\left[(\mathrm{c})\right.$ and (d)] with incoming neutron energy of $E_{i}=12 \mathrm{meV}$ [(a) and (c)] and $41 \mathrm{meV}$ [(b) and (d)]. In (a) and (c) [(b) and (d)], data points represent intensities integrated within a momentum transfer range of $1.2 \sim 2.2(2.2 \sim 3.2) \AA^{-1}$. Solid lines are the corresponding fits using the CEF Hamiltonian plus a polynomial background term shown as the dashed lines. Error bars representing standard deviations are smaller than the symbol size. The fitted CEF parameters are shown in Table I.

their $Q$ dependence together with imperfect descriptions of the background using only the polynomial terms.

The obtained CEF ground state wave functions for the $\mathrm{Er}^{3+}$ ions are $| \pm\rangle= \pm 0.123| \pm 11 / 2\rangle+0.396 \mid \pm$ $5 / 2\rangle \pm 0.596|\mp 1 / 2\rangle-0.516|\mp 7 / 2\rangle \pm 0.453|\mp 13 / 2\rangle$. The anisotropic $g$ factors are $g_{\perp}=7.8$ in the $x y$ plane and $g_{\|}=$ 4.7 along the $z$ direction, which sharply contrasts the Ising anisotropy observed in $\operatorname{CdEr}_{2} X_{4}(X=\mathrm{S}, \mathrm{Se})$ [42]. Such a difference originates from the different components of the CEF ground states: In $\mathrm{CdEr}_{2} X_{4}(X=\mathrm{S}, \mathrm{Se})$, the ground states are dominated by the $| \pm 15 / 2\rangle$ components, while in $\mathrm{NaErS}_{2}$, the largest components are $| \pm 1 / 2\rangle$. In the latter case, substantial quantum tunneling can be expected, which will facilitate the development of the QSL state.

\section{DISCUSSIONS}

The existence of the large $\left|J_{z}= \pm 1 / 2\right\rangle$ components in the $\mathrm{Er}^{3+}$ ground state wave functions can be understood through the point charge calculations. Assuming point charges of $-2 e$ on the surrounding sulfur anion sites, the calculated CEF ground state doublet will be dominated by the $| \pm 15 / 2\rangle$ components with $g_{\perp}=0$ and $g_{\|}=17.8$. However, once the additional $+3 e$ charges on the neighboring $\mathrm{Er}$ sites within the $a b$ plane are considered, the ground state wave functions will become $| \pm\rangle=0.004| \pm 13 / 2\rangle \pm 0.136| \pm 7 / 2\rangle+$ $0.981| \pm 1 / 2\rangle \mp 0.136|\mp 5 / 2\rangle+0.002|\mp 11 / 2\rangle$ with $g_{\perp}=$ 9.6 and $g_{\|}=1.2$, which is qualitatively similar to the results obtained from the INS spectra. Therefore, the electric charges beyond the $\mathrm{ErS}_{6}$ octahedra play an important role in deter-
TABLE II. The Wybourne CEF parameters (meV) for different rare earth ions.

\begin{tabular}{lcccccc}
\hline \hline & $L_{2}^{0}$ & $L_{4}^{0}$ & $L_{4}^{3}$ & $L_{6}^{0}$ & $L_{6}^{3}$ & $L_{6}^{6}$ \\
\hline $\mathrm{Nd}^{3+}$ & -38.7 & -176.0 & -293.2 & 91.4 & -0.4 & 76.9 \\
$\mathrm{Sm}^{3+}$ & -33.8 & -136.7 & -227.7 & - & - & - \\
$\mathrm{Tb}^{3+}$ & -28.5 & -99.8 & -166.3 & 41.7 & -0.2 & 35.0 \\
$\mathrm{Dy}^{3+}$ & -27.1 & -91.0 & -151.6 & 36.8 & -0.1 & 30.9 \\
$\mathrm{Ho}^{3+}$ & -25.8 & -83.4 & -138.9 & 32.7 & -0.1 & 27.5 \\
$\mathrm{Tm}^{3+}$ & -23.6 & -71.0 & -118.2 & 26.4 & -0.1 & 22.2 \\
$\mathrm{Yb}^{3+}$ & -22.6 & -65.9 & -109.7 & 23.9 & -0.1 & 20.1 \\
\hline \hline
\end{tabular}

mining the $\mathrm{Er}^{3+}$ ground state properties as for the $\mathrm{Yb}^{3+}$ ions in $\mathrm{YbMgGaO}_{4}$ [54].

The CEF parameters determined for $\mathrm{Er}^{3+}$ in $\mathrm{NaErS}_{2}$ can be scaled to other rare earth ions, thus providing basic knowledge on the CEF ground state in the sulfide delafossites. For this purpose, we first calculated the Hutchings CEF parameters for the $\mathrm{Er}^{3+}$ ions [49]. The Hutchings CEF parameters depend only on the CEF environment and can be conveniently applied for systems with similar crystal structures [41,42]. Assuming the same Hutchings CEF parameters, Table II lists the corresponding Wybourne CEF parameters for different rare earth ions. $\mathrm{Ce}^{3+}$ and $\mathrm{Pr}^{3+}$ are omitted because the corresponding $\mathrm{NaLnS}_{2}$ compounds do not crystallize in the $R \overline{3} \mathrm{~m}$ space group [31]. For the Kramers ions, the CEF ground state doublet can be calculated as follows:

$$
\begin{aligned}
\mathrm{Nd}^{3+}(J=9 / 2)| \pm\rangle= & 0.346| \pm 7 / 2\rangle \pm 0.122| \pm 1 / 2\rangle \\
& +0.930|\mp 5 / 2\rangle, \\
\mathrm{Sm}^{3+}(J=5 / 2)| \pm\rangle= & 0.806| \pm 5 / 2\rangle \pm 0.592|\mp 1 / 2\rangle, \\
\mathrm{Dy}^{3+}(J=15 / 2)| \pm\rangle= & 0.318| \pm 13 / 2\rangle \mp 0.504| \pm 7 / 2\rangle \\
& +0.325| \pm 1 / 2\rangle \pm 0.536|\mp 5 / 2\rangle \\
& +0.501|\mp 11 / 2\rangle, \\
\mathrm{Yb}^{3+}(J=7 / 2)| \pm\rangle= & 0.484| \pm 7 / 2\rangle \mp 0.525| \pm 1 / 2\rangle \\
& -0.700|\mp 5 / 2\rangle .
\end{aligned}
$$

Sizable $J_{z}= \pm 1 / 2$ components in the ground state doublet are predicted for all the Kramers ions, which supports the delafossites as candidate compounds for QSL states. Especially, in the case of $\mathrm{Yb}^{3+}$, the scaled CEF parameters predict three excitations from the CEF ground state at 21.4, 30.0 , and $55.8 \mathrm{meV}$ with a cross section of 5.0, 4.0, and 0.1 barn, respectively. This calculation result is close to the experimental observation of two CEF transitions at 23 and $39 \mathrm{meV}$ [35]. Although the exact crystallographic structure and consequently the Hutchings CEF parameters depend on the rare earth ions, we expect the scaled CEF ground state wave function to be qualitatively correct $[41,42]$, which supports the ternary sulfide delafossites as candidate compounds to realize the QSL state.

\section{CONCLUSIONS}

INS experiments have been performed on the QSL candidate $\mathrm{NaErS}_{2}$ to study the $\mathrm{Er}^{3+} \mathrm{CEF}$ transitions. The measured 
INS spectra can be fitted with the CEF Hamiltonian, which reveals the existence of large $J_{z}= \pm 1 / 2$ components in the ground state doublet that allows quantum fluctuations. Applying the fitted CEF parameters to other rare earth ions reveals that the $J_{z}= \pm 1 / 2$ components also exist in the CEF ground states, supporting the rare-earth-based sulfide delafossites as candidate hosts for the QSL state.

Note added. The recent publication [55] on $\mathrm{KErS}_{2}$ and $\mathrm{CsErS}_{2}$ reveals similar CEF parameter and ground state wave functions as in our works.

\section{ACKNOWLEDGMENTS}

We thank T. Nakajima, M. Soda, L. Ding, and V. Kocsis for helpful discussions. Our inelastic neutron scattering experiment was performed at the Materials and Life Science Experimental Facility (MLF) of the Japan Proton Accelerator Research Complex (J-PARC) under the user program (Proposal No. 2019A0293). F.X. acknowledges the funding from the European Union's Horizon 2020 research and innovation program under the Marie Skłodowska-Curie Grant Agreement No. 701647.
[1] P. Fazekas and P. W. Anderson, Philos. Mag. 30, 423 (1974).

[2] T. Giamarchi, Quantum Physics in One Dimension (Oxford Science Publications, Oxford, 2003).

[3] B. Normand, Contemp. Phys. 50, 533 (2009).

[4] L. Balents, Nature (London) 464, 199 (2010).

[5] L. Savary and L. Balents, Rep. Prog. Phys. 80, 016502 (2016).

[6] Y. Zhou, K. Kanoda, and T.-K. Ng, Rev. Mod. Phys. 89, 025003 (2017).

[7] A. Kitaev, Ann. Phys. 321, 2 (2006).

[8] C. Nayak, S. H. Simon, A. Stern, M. Freedman, and S. Das Sarma, Rev. Mod. Phys. 80, 1083 (2008).

[9] Y. Shimizu, K. Miyagawa, K. Kanoda, M. Maesato, and G. Saito, Phys. Rev. Lett. 91, 107001 (2003).

[10] P. Mendels, F. Bert, M. A. de Vries, A. Olariu, A. Harrison, F. Duc, J. C. Trombe, J. S. Lord, A. Amato, and C. Baines, Phys. Rev. Lett. 98, 077204 (2007).

[11] T.-H. Han, J. S. Helton, S. Chu, D. G. Nocera, J. A. RodriguezRivera, C. Broholm, and Y. S. Lee, Nature (London) 492, 406 (2012).

[12] M. Fu, T. Imai, T.-H. Han, and Y. S. Lee, Science 350, 655 (2015).

[13] J. G. Rau and M. J. P. Gingras, Phys. Rev. B 92, 144417 (2015).

[14] J. G. Rau and M. J. P. Gingras, Phys. Rev. B 98, 054408 (2018).

[15] M. Hermele, M. P. A. Fisher, and L. Balents, Phys. Rev. B 69, 64404 (2004).

[16] M. J. P. Gingras and P. A. McClarty, Rep. Prog. Phys. 77, 056501 (2014).

[17] S. T. Bramwell and M. J. P. Gingras, Science 294, 1495 (2001).

[18] T. Fennell, P. P. Deen, A. R. Wildes, K. Schmalzl, D. Prabhakaran, A. T. Boothroyd, R. J. Aldus, D. F. McMorrow, and S. T. Bramwell, Science 326, 415 (2009).

[19] T. Fennell, M. Kenzelmann, B. Roessli, M. K. Haas, and R. J. Cava, Phys. Rev. Lett. 109, 17201 (2012).

[20] L.-J. Chang, S. Onoda, Y. Su, Y.-J. Kao, K.-D. Tsuei, Y. Yasui, K. Kakurai, and M. R. Lees, Nat. Commun. 3, 992 (2012).

[21] R. Sibille, N. Gauthier, H. Yan, M. Ciomaga Hatnean, J. Ollivier, B. Winn, U. Filges, G. Balakrishnan, M. Kenzelmann, N. Shannon, and T. Fennell, Nat. Phys. 14, 711 (2018).

[22] O. Benton, O. Sikora, and N. Shannon, Phys. Rev. B 86, 075154 (2012).

[23] Y.-D. Li, X. Wang, and G. Chen, Phys. Rev. B 94, 035107 (2016).

[24] Y. Li, G. Chen, W. Tong, L. Pi, J. Liu, Z. Yang, X. Wang, and Q. Zhang, Phys. Rev. Lett. 115, 167203 (2015).
[25] J. A. M. Paddison, M. Daum, Z. Dun, G. Ehlers, Y. Liu, M. B. Stone, H. Zhou, and M. Mourigal, Nat. Phys. 13, 117 (2016).

[26] Y. Shen, Y.-D. Li, H. Wo, Y. Li, S. Shen, B. Pan, Q. Wang, H. C. Walker, P. Steffens, M. Boehm, Y. Hao, D. L. Quintero-Castro, L. W. Harriger, M. D. Frontzek, L. Hao, S. Meng, Q. Zhang, G. Chen, and J. Zhao, Nature (London) 540, 559 (2016).

[27] Y. Xu, J. Zhang, Y. S. Li, Y. J. Yu, X. C. Hong, Q. M. Zhang, and S. Y. Li, Phys. Rev. Lett. 117, 267202 (2016).

[28] Z. Zhu, P. A. Maksimov, S. R. White, and A. L. Chernyshev, Phys. Rev. Lett. 119, 157201 (2017).

[29] I. Kimchi, A. Nahum, and T. Senthil, Phys. Rev. X 8, 031028 (2018).

[30] T. Schleid and F. Lissner, Eur. J. Solid State Inorg. Chem. 30, 829 (1993).

[31] W. Liu, Z. Zhang, J. Ji, Y. Liu, J. Li, X. Wang, H. Lei, G. Chen, and Q. Zhang, Chin. Phys. Lett. 35, 117501 (2018).

[32] Y. Hashimoto, M. Wakeshima, and Y. Hinatsu, J. Solid State Chem. 176, 266 (2003).

[33] B. Dong, Y. Doi, and Y. Hinatsu, J. Alloys Compd. 453, 282 (2008).

[34] N. Miyasaka, Y. Doi, and Y. Hinatsu, J. Solid State Chem. 182, 2104 (2009).

[35] M. Baenitz, P. Schlender, J. Sichelschmidt, Y. A. Onykiienko, Z. Zangeneh, K. M. Ranjith, R. Sarkar, L. Hozoi, H. C. Walker, J.-C. Orain, H. Yasuoka, J. van den Brink, H. H. Klauss, D. S. Inosov, and T. Doert, Phys. Rev. B 98, 220409(R) (2018).

[36] J. Sichelschmidt, P. Schlender, B. Schmidt, M. Baenitz, and T. Doert, J. Phys.: Condens. Matter 31, 205601 (2019).

[37] M. M. Bordelon, E. Kenney, C. Liu, T. Hogan, L. Posthuma, M. Kavand, Y. Lyu, M. Sherwin, N. P. Butch, C. Brown, M. J. Graf, L. Balents, and S. D. Wilson, Nat. Phys. 15, 1058 (2019).

[38] K. M. Ranjith, D. Dmytriieva, S. Khim, J. Sichelschmidt, S. Luther, D. Ehlers, H. Yasuoka, J. Wosnitza, A. A. Tsirlin, H. Kühne, and M. Baenitz, Phys. Rev. B 99, 180401(R) (2019).

[39] L. Ding, P. Manuel, S. Bachus, F. Grußler, P. Gegenwart, J. Singleton, R. D. Johnson, H. C. Walker, D. T. Adroja, A. D. Hillier, and A. A. Tsirlin, Phys. Rev. B 100, 144432 (2019).

[40] J. S. Gardner, M. J. P. Gingras, and J. E. Greedan, Rev. Mod. Phys. 82, 53 (2010).

[41] A. Bertin, Y. Chapuis, P. Dalmas de Réotier, and A. Yaouanc, J. Phys.: Condens. Matter 24, 256003 (2012).

[42] S. Gao, O. Zaharko, V. Tsurkan, L. Prodan, E. Riordan, J. Lago, B. Fåk, A. R. Wildes, M. M. Koza, C. Ritter, P. Fouquet, L. Keller, E. Canévet, M. Medarde, J. Blomgren, C. Johansson, S. R. Giblin, S. Vrtnik, J. Luzar, A. Loidl, C. Rüegg, and T. Fennell, Phys. Rev. Lett. 120, 137201 (2018). 
[43] D. Reig-i Plessis, A. Cote, S. van Geldern, R. D. Mayrhofer, A. A. Aczel, and G. J. MacDougall, Phys. Rev. Mater. 3, 114408 (2019).

[44] J. Rodriguez-Carvajal, Physica B: Condens. Matter 192, 55 (1993).

[45] R. Kajimoto, M. Nakamura, Y. Inamura, F. Mizuno, K. Nakajima, S. Ohira-Kawamura, T. Yokoo, T. Nakatani, R. Maruyama, K. Soyama, K. Shibata, K. Suzuya, S. Sato, K. Aizawa, M. Arai, S. Wakimoto, M. Ishikado, S.-i. Shamoto, M. Fujita, H. Hiraka, K. Ohoyama, K. Yamada, and C.-H. Lee, J. Phys. Soc. Jpn. 80, SB025 (2011).

[46] M. Ruminy, E. Pomjakushina, K. Iida, K. Kamazawa, D. T. Adroja, U. Stuhr, and T. Fennell, Phys. Rev. B 94, 024430 (2016).

[47] M. Nakamura, R. Kajimoto, Y. Inamura, F. Mizuno, M. Fujita, T. Yokoo, and M. Arai, J. Phys. Soc. Jpn. 78, 093002 (2009).
[48] Y. Inamura, T. Nakatani, J. Suzuki, and T. Otomo, J. Phys. Soc. Jpn. 82, SA031 (2013).

[49] M. Hutchings, Solid State Phys. 16, 227 (1964).

[50] M. Rotter, J. Mag. Magn. Mater. 272, E481 (2004).

[51] S. Rosenkranz, A. P. Ramirez, A. Hayashi, R. J. Cava, R. Siddharthan, and B. S. Shastry, J. Appl. Phys. 87, 5914 (2000).

[52] J. Xu, V. K. Anand, A. K. Bera, M. Frontzek, D. L. Abernathy, N. Casati, K. Siemensmeyer, and B. Lake, Phys. Rev. B 92, 224430 (2015).

[53] M. D. Le, http://yocto.me/work/saficf/index.html.

[54] Y. Li, D. Adroja, R. I. Bewley, D. Voneshen, A. A. Tsirlin, P. Gegenwart, and Q. Zhang, Phys. Rev. Lett. 118, 107202 (2017).

[55] A. Scheie, V. O. Garlea, L. D. Sanjeewa, J. Xing, and A. S. Sefat, Phys. Rev. B 101, 144432 (2020). 